\title{
ON INDUCED CHARACTERS
}

\author{
YAKOV BERKOVICH
}

(Communicated by Ronald M. Solomon)

\begin{abstract}
Suppose that $H$ is a normal subgroup of a finite group $G, \varphi \in$ $\operatorname{Irr}(H)$, and $\operatorname{Irr}\left(\varphi^{G}\right)$ is the set of all irreducible constituents of the induced character $\varphi^{G}$. If $\left|\operatorname{Irr}\left(\varphi^{G}\right)\right|>|G: H| / 4$ then $G / H$ is solvable.
\end{abstract}

If $\tau$ is a character of a group, then by $\operatorname{Irr}(\tau)$ we denote the set of all irreducible constituents of $\tau$. Set $s(\tau)=|\operatorname{Irr}(\tau)|$ and $w(\tau)=\sum\langle\tau, \chi\rangle$, where $\chi$ runs over the set $\operatorname{Irr}(G)$ of all irreducible characters of $G$. Obviously $s(\tau) \leq w(\tau)$.

In this note we prove the following

Theorem. Suppose that $H$ is a proper normal subgroup of a finite group $G, p$ is the smallest prime dividing $|G: H|$, and $\varphi$ is an irreducible character of $H$.

(a) If $s\left(\varphi^{G}\right) \geq|G: H| / p^{2}$ then $G / H$ is solvable unless $\varphi$ is G-invariant, $\varphi^{G}=p\left(\chi^{1}+\cdots+\chi^{s}\right)$, where $\operatorname{Irr}\left(\varphi^{G}\right)=\left\{\chi^{1}, \ldots, \chi^{s}\right\}, s\left(\varphi^{G}\right)=$ $|G: H| / p^{2}$.

(b) If $w\left(\varphi^{G}\right) \geq|G: H| / p$ then $G / H$ is solvable unless the same exception holds as in (a).

We consider only finite groups.

A group $G$ is said to be $p$-nilpotent ( $p$ is always a prime) if it has a normal $p$-complement. A group $G$ is said to be dispersive if its arbitrary subgroup $A$ is $p$-nilpotent for the smallest prime $p$ dividing $|A|$.

We fix the following notation. Let $\operatorname{Irr}(G)=\left\{\chi^{1}, \ldots, \chi^{k}\right\}$, where $k=k(G)$ is the class number of $G$. The number $\operatorname{mc}(G)=k(G) /|G|$ is called the measure of commutativity of $G$. Obviously $0<\operatorname{mc}(G) \leq 1$ and $\operatorname{mc}(G)=1$ iff $G$ is abelian. Denote by $T(G)$ the sum of degrees of all irreducible characters of $G$ and set $f(G)=T(G) /|G|$. Note that $G$ is abelian iff $f(G)=1$.

Lemma 1 [7]. Let $H$ be a subgroup of a group $G$. Then:

(a) $\mathrm{mc}(H) \geq \mathrm{mc}(G)$.

(b) $f(H) \geq f(G)$.

(c) If $H$ is normal in $G$ then $\mathrm{mc}(G / H) \geq \operatorname{mc}(G)$.

(d) $\mathrm{mc}(G) \geq f(G)^{2} ; \operatorname{mc}(G)=f(G)^{2}$ iff $G$ is abelian.

Received by the editors February 1, 1992 and, in revised form, October 25, 1992.

1991 Mathematics Subject Classification. Primary 20C15, 20 D20.

The author was supported in part by the Ministry of Absorption and the Rashi Foundation. 
Proof. (a) By reciprocity

$$
k(G)=|\operatorname{Irr}(G)| \leq \sum_{\psi \in \operatorname{Irr}(H)}\left|\operatorname{Irr}\left(\psi^{G}\right)\right| \leq|G: H||\operatorname{Irr}(H)|=|G: H| k(H)
$$

and

$$
\operatorname{mc}(G)=k(G) /|G| \leq k(H) /|H|=\operatorname{mc}(H) .
$$

(b) By reciprocity

$$
T(G)=\sum_{\chi \in \operatorname{Irr}(G)} \chi(1) \leq \sum_{\psi \in \operatorname{Irr}(H)} \psi^{G}(1)=|G: H| \sum_{\psi \in \operatorname{Irr}(H)} \psi(1)=|G: H| T(H)
$$

and

$$
f(G)=T(G) /|G| \leq T(H) /|H|=f(H) .
$$

(c) Let $k_{G}(M)$ denote the number of $G$-classes (= classes of conjugate elements of $G$ ) having nonempty intersections with the subset $M$ of $G$. For $x \in G$ denote by $K(x)$ the $G$-class containing $x$. Obviously $k_{G}(K(x) H)=$ $k_{G}(x H) \leq|H|$. Obviously there exists a subset $\mathfrak{M} \subseteq G$ such that

$$
G=\sum_{x \in \mathfrak{M}} K(x) H
$$

is a partition. Since $|\mathfrak{M}|=k(G / H)$ then

$$
k(G)=\sum_{x \in \mathfrak{M}} k_{G}(K(x) H) \leq|\mathfrak{M}||H|=k(G / H)|H|
$$

so that

$$
\operatorname{mc}(G)=k(G) /|G| \leq k(G / H)|H| /|G|=\operatorname{mc}(G / H) .
$$

(d) (Mann) Consider two $k$-dimensional vectors $(k=k(G))$

$$
\mathbf{a}=\left(\chi^{1}(1), \ldots, \chi^{k}(1)\right), \quad \mathbf{b}=(1, \ldots, 1) .
$$

Then by the Cauchy-Schwartz inequality

$$
(|G| f(G))^{2}=T(G)^{2}=(\mathbf{a} \cdot \mathbf{b})^{2} \leq\left\|\mathbf { a } \left|\left\|\left.|\mathbf{b} \|=| G|k(G)=| G\right|^{2} \operatorname{mc}(G)\right.\right.\right.
$$

and our inequality follows. If $f(G)^{2}=\operatorname{mc}(G)$ then vectors a and b are linearly dependent. In this case $\chi^{1}(1)=\cdots=\chi^{k}(1)=1$ and $G$ is abelian.

We note that Lemma $1(c)$ is a consequence of the inequality

$$
k(G) \leq k(G / H) k(H)
$$

which is due to Gallagher. For the other proof of Lemma 1(d) see [7].

Lemma 2. Suppose that $G=R G^{\prime}$ is a Frobenius group with the kernel $G^{\prime}$ and a complementary factor $R,|R|=q$ is a prime, $G^{\prime}$, the commutator subgroup of $G$, is an elementary abelian group of order $p^{b}$, and $p$ is a prime.

(a) If $r=\min \{p, q\}$ then $\operatorname{mc}(G)<(r+1) / r^{2}$.

(b) If $p<q$ then $\operatorname{mc}(G)<1 / p^{2}$ unless $G \cong A_{4}$, the alternating group of degree 4. 
Proof. One has

$$
\operatorname{mc}(G)=q^{-2} p^{-b}\left(p^{b}-1+q^{2}\right) .
$$

(a) Suppose that $\operatorname{mc}(G) \geq(r+1) / r^{2}$.

(1a) Let $p<q$. Then $b>1$ and

$$
p^{b}-1+q^{2} \geq q^{2} p^{b-2}(p+1)=q^{2} p^{b-1}+q^{2} p^{b-2}
$$

which is impossible.

(2a) Let $q<p$. Then

$$
\begin{gathered}
p^{b}-1+q^{2} \geq(q+1) p^{b}=q p^{b}+p^{b}, \\
q^{2}-1 \geq q p^{b} \geq q(q+1)=q^{2}+q,
\end{gathered}
$$

a contradiction, and (a) is proved.

(b) Suppose that $\operatorname{mc}(G) \geq 1 / p^{2}$. Then

$$
p^{b}-1+q^{2} \geq q^{2} p^{b-2} .
$$

If $b=2$ then $p=2, q=3$, and $G \cong A_{4}$. Let $b>2$. Then

$$
\begin{gathered}
\left(p^{b}-1\right) /\left(p^{b-2}-1\right) \geq q^{2} \geq(p+1)^{2}, \\
p^{b-1} \geq\left(p^{b-2}-1\right)(p+1)^{2}=p^{b-2}(p+1)^{2}-(p+1)^{2} \\
=p^{b}+2 p^{b-1}+p^{b-2}-(p+1)^{2}, \\
(p+1)^{2} \geq 2 p^{b-1}+p^{b-2}+1 \geq 2 p^{2}+p+1,
\end{gathered}
$$

a contradiction.

Lemma 3. Suppose that $p$ is the smallest prime divisor of the order of a group $G$.

(a) If $\mathrm{mc}(G) \geq(p+1) / p^{2}$ then $G$ is abelian.

(b) If $\operatorname{mc}(G) \geq 1 / p^{2}$ then $G$ is solvable, and $G$ is dispersive if $p>2$.

(c) If $f(G) \geq 1 / p$ then $G$ is dispersive unless $\left|G^{\prime}\right| \in\left\{2^{2}, 2^{3}\right\}$.

Proof. (a) Suppose that $G$ is a counterexample of minimal order. Then (Lemma $1(a)$, (c)) $G$ is a minimal nonabelian group. By the Miller-Moreno Theorem [6] one of the following assertions holds:

(i) $|G|=p^{n},\left|G^{\prime}\right|=p,|G: Z(G)|=p^{2}$.

(ii) $G=Q G^{\prime}$, a semidirect product of $Q, G^{\prime} \in \operatorname{Syl}(G), G^{\prime}$ is elementary abelian.

If (i) holds one obtains

$$
\begin{aligned}
\operatorname{mc}(G) & =p^{-n} k(G)=p^{-n}\left(p^{n-2}+p^{n-1}-p^{n-3}\right) \\
& =p^{-2}(p+1)-p^{-3}<p^{-2}(p+1),
\end{aligned}
$$

a contradiction.

If (ii) holds one obtains $\mathrm{mc}(G) \leq \mathrm{mc}(G / Z(G))$, and a contradiction follows from Lemma 2(a).

(b) At first we prove that $G$ is solvable. Suppose that $G$ is a counterexample of minimal order. Then all proper subgroups and epimorphic images of $G$ are solvable, but $G$ is nonsolvable (Lemma $1(\mathrm{a})$, (c)). So $G$ is a nonabelian simple 
group. Suppose that $\chi^{1}(1) \leq \cdots \leq \chi^{k}(1)$. Then $\chi^{i}(1) \geq p$ for $i>1$ and $\chi^{k}(1) \geq p+1[5$, Theorem 6.9]. Hence

$$
\begin{aligned}
|G| & =1+\sum_{i=2}^{k-1} \chi^{i}(1)^{2}+\chi^{k}(1)^{2} \geq 1+(k-2) p^{2}+(p+1)^{2} \\
& =(k-1) p^{2}+2 p+2 \geq\left(|G| / p^{2}-1\right) p^{2}+2 p+2 \\
& =|G|+2 p+2-p^{2} .
\end{aligned}
$$

Hence if $p=2$ then we have $|G| \geq|G|+2$, a contradiction. Suppose that $p>2$ and prove that $G$ is dispersive. In view of Lemma $1(a)$ it is sufficient to prove that $G$ is $p$-nilpotent. Suppose that $G$ is a counterexample of minimal order. Then $G$ is a minimal nonnilpotent group with a normal Sylow $p$-subgroup [4, Satz 4.5.4]. Then (Lemmas $1(\mathrm{c})$ and $2(\mathrm{~b})$ ) one has

$$
\operatorname{mc}(G) \leq \operatorname{mc}(G / Z(G))<1 / p^{2}
$$

a contradiction.

(c) is proved in [7].

Proof of the Theorem. (a) At first suppose that $H=1$. Without loss of generality we may assume that $\varphi=1_{H}$. Then $\varphi^{G}=\rho_{G}$, the regular character of $G, \operatorname{Irr}\left(\varphi^{G}\right)=\operatorname{Irr}(G)=\left\{\chi^{1}, \ldots, \chi^{k}\right\}$, where $k=k(G)$, the class number of $G$. In our case $s\left(\varphi^{G}\right)=k(G)$. Therefore by the condition $k(G) \geq|G| / p^{2}$, $\operatorname{mc}(G) \geq 1 / p^{2}$, and $G$ is solvable (Lemma $3(\mathrm{~b})$ ).

Suppose that $H>1$. Let

$$
\operatorname{Irr}\left(\varphi^{G}\right)=\left\{\chi^{1}, \ldots, \chi^{s}\right\} \quad \text { and } \quad \varphi^{G}=e_{1} \chi^{1}+\cdots+e_{s} \chi^{s} .
$$

If

$$
\chi_{H}^{i}=e_{i}\left(\varphi_{1}+\cdots+\varphi_{t}\right)
$$

is the Clifford decomposition, $\varphi_{1}=\varphi, t=\left|G: I_{G}(\varphi)\right|$, where $I_{G}(\varphi)$ is the inertia group of $\varphi$ in $G$, then $\chi^{i}(1)=e_{i} t \varphi(1)$ for all $i$ and

$$
\begin{gathered}
|G: H| \varphi(1)=\varphi^{G}(1)=t \varphi(1)\left(e_{1}^{2}+\cdots+e_{s}^{2}\right), \\
\left|I_{G}(\varphi): H\right|=e_{1}^{2}+\cdots+e_{s}^{2} .
\end{gathered}
$$

Since $s=s\left(\varphi^{G}\right) \geq|G: H| / p^{2}$, we have

$$
|G: H|=t\left(e_{1}^{2}+\cdots+e_{s}^{2}\right) \geq t s \geq t|G: H| / p^{2} ;
$$

then $t \leq p^{2}$. Since $t$ is a divisor of $|G: H|$ then $t \in\left\{1, p^{2}, q\right\}$, where $q$ is a prime (we recall that $p$ is the smallest prime divisor of $|G: H|$ ).

(i) Suppose that $t=p^{2}$. Then $e_{1}=\cdots=e_{s}=1$ by $(*)$. By [5, Theorem 6.11] we have

$$
\begin{gathered}
\operatorname{Irr}\left(\varphi^{I_{G}(\varphi)}\right)=\left\{\psi_{1}, \ldots, \psi_{s}\right\}, \\
\varphi^{I_{G}(\varphi)}=e_{1} \psi_{1}+\cdots+e_{s} \psi_{s}=\psi_{1}+\cdots+\psi_{s} .
\end{gathered}
$$

Then by reciprocity $\left(\psi_{1}\right)_{H}=\varphi$ and (by Gallagher's Theorem [5, Corollary 6.17]) $\left|\operatorname{Irr}\left(I_{G}(\varphi) / H\right)\right|=s, \operatorname{Irr}\left(I_{G}(\varphi) / H\right)=\left\{\beta_{1}, \ldots, \beta_{s}\right\}, \beta_{i}(1)=e_{i}$, and then $\psi_{i}=\psi_{1} \beta_{i}$ (after possible reordering) for $i=1, \ldots, s$. Now

$$
\operatorname{mc}\left(I_{G}(\varphi) / H\right)=s\left|I_{G}(\varphi) / H\right|^{-1} \geq\left(|G: H| / p^{2}\right)(|G: H| / t)^{-1}=t / p^{2}=1,
$$


and $I_{G}(\varphi) / H$ is abelian. Then $G / H$ is solvable as a product of $I_{G}(\varphi) / H$ and the Sylow $p$-subgroup of $G / H$ (see, for example, [4, Satz 6.4.11]).

(ii) Suppose that $t$ is a prime. Then $I_{G}(\varphi) / H$ is maximal in $G / H$. By [5, Theorem 6.11] the equalities $(* *)$ are true.

Suppose that all $e_{i}>1$. Then all $e_{i} \geq p$ since $e_{1}, \ldots, e_{s}$ as degrees of irreducible projective representations of $I_{G}(\varphi) / H$ are divisors of $\left|I_{G}(\varphi) / H\right|$. Hence

$$
\begin{aligned}
\left|I_{G}(\varphi) / H\right| & =e_{1}^{2}+\cdots+e_{s}^{2} \geq p^{2} s \geq p^{2}\left(|G: H| / p^{2}\right) \\
& =|G: H|=t\left|I_{G}(\varphi) / H\right|>\left|I_{G}(\varphi) / H\right|,
\end{aligned}
$$

a contradiction. Supposing $e_{1} \leq \cdots \leq e_{S}$, we have $e_{1}=1$. The $\left(\psi_{1}\right)_{H}=\varphi$ and, as in (i), using [5, Corollary 6.17], we obtain $\operatorname{mc}\left(I_{G}(\varphi) / H\right) \geq t / p^{2}$. Then $I_{G}(\varphi) / H$ is solvable by Lemma $3(\mathrm{~b})$.

If $t=p$ then $I_{G}(\varphi) / H$ is normal in $G / H$, and $G / I_{G}(\varphi)$ is cyclic of order $p$. Therefore $G / H$ is solvable in this case.

Suppose that $t>p$. Then

$$
\operatorname{mc}\left(I_{G}(\varphi) / H\right) \geq(p+1) / p^{2}
$$

and $I_{G}(\varphi) / H$ is abelian by Lemma $3($ a), so that $G / H$ is solvable by Herstein's Theorem [3].

(iii) Suppose that $t=1$. Then $\varphi$ is $G$-invariant. If $e_{i}=1$ for some $i \in\{1, \ldots, s\}$ then as above $G / H$ is solvable. Suppose that all $e_{i}>1$. Then all $e_{i} \geq p$ and

$$
|G: H|=e_{1}^{2}+\cdots+e_{s}^{2} \geq s p^{2} \geq\left(|G: H| / p^{2}\right) p^{2}=|G: H| .
$$

Hence $s=|G: H| / p^{2}, e_{1}=\cdots=e_{s}=p$, and assertion (a) is proved.

(b) Suppose that $H=1$. Without loss of generality we may assume that $\varphi=1_{H}$. Then $\varphi^{G}=\rho_{G}$, the regular character of $G$, and $|G| / p \leq w\left(\varphi^{G}\right)=$ $T(G)=|G| f(G)$ and $G$ is solvable by Lemma $3(\mathrm{c})$.

Let $H>1$. Let as before

$$
\operatorname{Irr}\left(\varphi^{G}\right)=\left\{\chi^{1}, \ldots, \chi^{s}\right\}, \quad \varphi^{G}=e_{1} \chi^{1}+\cdots+e_{s} \chi^{s} .
$$

Then

$$
w\left(\varphi^{G}\right)=e_{1}+\cdots+e_{s} \geq|G: H| / p
$$

As before one has

$$
|G: H|=t\left(e_{1}^{2}+\cdots+e_{s}^{2}\right), \quad t=\left|G: I_{G}(\varphi)\right| .
$$

Therefore

$$
\begin{aligned}
\left|I_{G}(\varphi): H\right| & =e_{1}^{2}+\cdots+e_{s}^{2} \geq e_{1}+\cdots+e_{s} \\
& =w\left(\varphi^{G}\right) \geq|G: H| / p=t\left|I_{G}(\varphi): H\right| / p \Rightarrow t \leq p .
\end{aligned}
$$

So $I_{G}(\varphi)$ is normal in $G$ and $G / I_{G}(\varphi)$ is cyclic.

Suppose that $e_{i}=1$ for some $i \in\{1, \ldots, s\}$. Then as in (a) one has

$$
\begin{aligned}
\left|I_{G}(\varphi) / H\right| f\left(I_{G}(\varphi) / H\right) & =T\left(I_{G}(\varphi) / H\right)=e_{1}+\cdots+e_{s}=w\left(\varphi^{G}\right) \geq|G: H| / p \\
& \left.=t \mid I_{G}(\varphi) / H\right) \mid / p \Rightarrow f\left(I_{G}(\varphi) / H\right) \geq 1 / p
\end{aligned}
$$

and $I_{G}(\varphi) / H$ is solvable (Lemma $3(\mathrm{c})$ ). Since $G / I_{G}(\varphi)$ is cyclic then $G / H$ is solvable. 
Suppose that all $e_{i}>1$. Then $e_{i} \geq p$ for all $i$. Therefore

$$
\begin{aligned}
\left|I_{G}(\varphi): H\right| & =e_{1}^{2}+\cdots+e_{s}^{2} \geq p\left(e_{1}+\cdots+e_{s}\right)=p w\left(\varphi^{G}\right) \geq p(|G: H| / p) \\
& =|G: H| \Rightarrow I_{G}(\varphi)=G, \quad e_{1}=\cdots=e_{s}=p .
\end{aligned}
$$

Remark. If, in the Theorem, $\varphi$ is reducible then $G / H$ is solvable unless for any $\lambda \in \operatorname{Irr}(\varphi)$ one has $\lambda^{G}=p\left(\chi^{1}+\cdots+\chi^{s}\right), s=|G: H| / p^{2}$, and $\operatorname{Irr}\left(\lambda^{G}\right)=$ $\left\{\chi^{1}, \ldots, \chi^{s}\right\}$.

Corollary. Suppose that $H$ is a proper normal subgroup of a group $G$, and $p$ is the smallest prime dividing $|G: H|$.

(a) If $w\left(\varphi^{G}\right) \geq|G: H| / p$ for all nonlinear $\varphi \in \operatorname{Irr}(H)$, then $G / H$ is solvable or $H^{\prime}$ has a normal p-complement.

(b) If $s\left(\varphi^{G}\right) \geq|G: H| / p^{2}$ for all nonlinear $\varphi \in \operatorname{Irr}(H)$, then the same conclusion as in (a) holds.

Proof. Suppose that $G / H$ is nonsolvable. We may assume that $H$ is nonabelian (so that $\operatorname{Irr}(H)$ contains a nonlinear character). Then for any nonlinear $\varphi \in \operatorname{Irr}(H)$ we have (by the Theorem)

$$
\varphi^{G}=p\left(\chi^{1}+\cdots+\chi^{s}\right), \quad \operatorname{Irr}\left(\varphi^{G}\right)=\left\{\chi^{1}, \ldots, \chi^{s}\right\} .
$$

By reciprocity $p$ divides degrees of all irreducible constituents of $\varphi^{G}$. Let

$$
\operatorname{Irr}\left(G, p^{\prime}\right)=\{\chi \in \operatorname{Irr}(G) \mid \chi(1)>1 \text { and } p \text { does not divide } \chi(1)\}
$$

and let $G\left(p^{\prime}\right)$ be the intersection of kernels of all characters belonging to $\operatorname{Irr}\left(G, p^{\prime}\right)$. The subgroup $G\left(p^{\prime}\right)$ is $p$-nilpotent [2]. Take $\chi \in \operatorname{Irr}\left(G, p^{\prime}\right)$. Then by the above all irreducible constituents of $\chi_{H}$ are linear so that $H^{\prime} \leq \operatorname{ker} \chi$. Therefore $H^{\prime} \leq G\left(p^{\prime}\right)$ and $H^{\prime}$ is $p$-nilpotent.

Remarks. 1. We note the crucial role of Gallagher's Theorem [5, Corollary 6.17] in the proof of the Theorem. Note that the assertion converse to Gallagher's Theorem is also true. Namely, if $N$ is a normal subgroup of $G$ and $\chi \in \operatorname{Irr}(G)$ then $\chi \theta \in \operatorname{Irr}(G)$ for all $\theta \in \operatorname{Irr}(G / N)$ implies $\chi_{N} \in \operatorname{Irr}(N)$. We prove this assertion. Take $\lambda \in \operatorname{Irr}\left(\chi_{N}\right)$. It is sufficient to prove that $\lambda(1)=\chi(1)$. Take $\psi \in \operatorname{Irr}(G / N)$. Then

$$
\left\langle\psi \chi, \lambda^{G}\right\rangle=\left\langle(\psi \chi)_{N}, \lambda\right\rangle=\psi(1)\left\langle\chi_{N}, \lambda\right\rangle
$$

so that $\psi(1) \psi \chi$ is a constituent of $\lambda^{G}$. Now

$$
\left(\chi_{N}\right)^{G}=\left(\chi_{N} \cdot 1_{N}\right)^{G}=\chi \rho_{G / N}
$$

where $\rho_{G / N}$ is the regular character of $G / N$. Put $\operatorname{Irr}(G / N)=\left\{\theta_{1}, \ldots, \theta_{n}\right\}$. Then

$$
\chi \rho_{G / N}=\sum_{i=1}^{n} \theta_{i}(1) \theta_{i} \chi
$$

by the above is a constituent of $\lambda^{G}$. Since

$$
\left(\chi \rho_{G / N}\right)(1)=|G: N| \chi(1) \geq|G: N| \lambda(1)=\lambda^{G}(1)
$$

then $\chi \rho_{G / N}=\lambda^{G}, \lambda(1)=\chi(1)$, and $\lambda=\chi_{N}$. Therefore $\chi_{N} \in \operatorname{Irr}(N)$ and our assertion is proved. 
2. If, in the Theorem,

$$
s\left(\varphi^{G}\right) \geq(p+1)|G: H| / p^{2}
$$

then $G / H$ is abelian. In particular if $s\left(\varphi^{G}\right)=|G: H|$ then $G / H$ is abelian.

3. Suppose that $H<G, \varphi \in \operatorname{Irr}(H)$, and $\chi \in \operatorname{Irr}(G)$. Then

$$
\begin{aligned}
& w\left(\varphi^{G}\right)>|G: H| / 2 \Rightarrow \min \left\{\left\langle\varphi^{G}, \tau\right\rangle \mid \tau \in \operatorname{Irr}\left(\varphi^{G}\right)\right\}=1, \\
& w\left(\chi_{H}\right)>|G: H| / 2 \Rightarrow \min \left\{\left\langle\chi_{H}, \psi\right\rangle \mid \psi \in \operatorname{Irr}\left(\chi_{H}\right)\right\}=1 .
\end{aligned}
$$

Analogous results hold for $s\left(\varphi^{G}\right)$ and $s\left(\chi_{H}\right)$.

4. Let $H<G$. If $s\left(\varphi^{G}\right)=|G: H|$ (or $w\left(\varphi^{G}\right)=|G: H|$ ) for all nonprincipal $\varphi \in \operatorname{Irr}(H)$ then $H$ is normal in $G$. We prove the first part of this assertion. If $\varphi \in \operatorname{Irr}(H)$ and $s\left(\varphi^{G}\right)=|G: H|$ then degrees of all irreducible constituents of $\varphi^{G}$ are equal to $\varphi(1)$. Take $\chi \in \operatorname{Irr}\left(\left(1_{H}\right)^{G}\right)$ and suppose that $\left|\operatorname{Irr}\left(\chi_{H}\right)\right|>1$. Take $\lambda \in \operatorname{Irr}\left(\chi_{H}\right)-\left\{1_{H}\right\}$. Since $s\left(\lambda^{G}\right)=|G: H|$ then by the above $\lambda(1)=\chi(1)$, a contradiction since $\lambda \in \operatorname{Irr}\left(\chi_{H}-1_{H}\right)$. So all irreducible constituents of $\left(1_{H}\right)^{G}$ are linear and $H$ is normal in $G$.

5. If $\operatorname{mc}(G)>1 / 12$ then $G$ is solvable [1]. So (Lemma $1(\mathrm{~d}))$ if $f(G)^{2}>$ $1 / 12$ then $G$ is solvable.

Conjectures. Suppose that $H<G$.

1. If $s\left(\varphi^{G}\right)>|G: H| / 4$ for all $\varphi \in \operatorname{Irr}(H)$ and $|G: H|$ is sufficiently large, then $H$ is normal in $G$.

2. If $w\left(\varphi^{G}\right)>|G: H| / 2$ for all $\varphi \in \operatorname{Irr}(H)$ and $|G: H|$ is sufficiently large, then $H$ is normal in $G$.

\section{ACKNOWLEDGMENTS}

I am indebted to Professors Emmanuel Zhmud' and Avinoam Mann for useful discussions. Also, my deep thanks to the referee for many useful remarks and suggestions.

\section{REFERENCES}

1. Ya. G. Berkovich, Relation between numbers of classes of a group and its subgroups, Questions of Group Theory and Homological Algebra, Jaroslavl, 1985, pp. 49-61. (Russian)

2. Degrees of irreducible characters and normal p-complements, Proc. Amer. Math. Soc. 106 (1989), 33-35.

3. I. N. Herstein, Remark on finite groups, Proc. Amer. Math. Soc. 9 (1958), 255-257.

4. B. Huppert, Endliche Gruppen, Bd. 1, Springer, Berlin, 1967.

5. I. M. Isaacs, Character theory of finite groups, Academic Press, New York, 1976.

6. G. A. Miller and H. Moreno, Non-abelian groups in which every subgroup is abelian, Trans. Amer. Math. Soc. 4 (1903), 398-404.

7. K. G. Nekrasov and Ya. G. Berkovich, Finite groups with large sums of degrees of irreducible characters, Publ. Math. Debrecen 33 (1986), 333-354. (Russian)

Department of Mathematics and Computer Science, Afula Research Institute, UniVERSITY OF HAIFA, 31905 HAIFA, ISRAEL 